PROCEEDINGS OF THE

AMERICAN MATHEMATICAL SOCIETY

Volume 125, Number 7, July 1997, Pages 2083-2092

S 0002-9939(97)03771-4

\title{
HARMONIC 2-SPHERES WITH $r$ PAIRS OF EXTRA EIGENFUNCTIONS
}

\author{
MOTOKO KOTANI
}

(Communicated by Peter Li)

\begin{abstract}
In the present paper, deformations of harmonic 2-spheres in the unit $n$-sphere $S^{n}$ respecting the degree are studied. The limit maps of such deformations are characterized as harmonic maps with extra eigenfunctions. The space $\operatorname{Harm}_{d}\left(S^{2}, S^{n}\right)$ of harmonic 2-spheres in $S^{n}$ with fixed degree $d$ is described in terms of such deformations and the limit maps.
\end{abstract}

\section{$\S 0$. INTRODUCTION}

Recently there has been much advance in the study of the space of all harmonic maps from a compact Riemann surface $\Sigma$ into a Riemann manifold $M$ as well as in study on individual harmonic maps. The space $\operatorname{Harm}_{d}\left(S^{2}, S^{n}\right)$ of harmonic maps from a compact Riemann surface $S^{2}$ of genus zero into the unit $n$-sphere $S^{n}$ with fixed degree $d$ has been shown to be path-connected if $n \geq 3$ ([V], [L] for $n=4,[\mathrm{~K}]$ for general $n \geq 3$ ) and its fundamental group is computed in [FGKO]. Similar results have been obtained for harmonic maps from $S^{2}$ to many other target manifolds. The space $\operatorname{Harm}_{d, e}\left(S^{2}, \mathbf{C} P^{N}\right)$ of harmonic maps into $\mathbf{C} P^{N}$ with fixed energy and degree for $N \geq 2\left([\mathrm{Cr}]\right.$, [GO]), the spaces $\operatorname{Harm}_{e}\left(S^{2}, \mathbf{H} P^{N}\right)^{\text {st.isot. }}$ and $\operatorname{Harm}_{e}\left(S^{2}, \mathbf{H} P^{N}\right)^{q u a t}$ of strongly isotropic harmonic maps and quaternionic mixed pairs, respectively, into $\mathbf{H} P^{N}$ with fixed energy ([M2], [GMO]), the space $\operatorname{Harm}_{d, q}\left(S^{2}, Q_{n}(\mathbf{C})\right)^{\text {st.isot }}$ of strongly isotropic harmonic maps into $Q_{n}(\mathbf{C})$ with fixed degree and energy ([M1], [GMO]), are all path-connected and the fundamental groups of some of them have been computed. Nevertheless, it seems that the structure of the space $\operatorname{Harm}_{d}\left(S^{2}, S^{n}\right)$, which is a fundamental object, still is not sufficiently well-understood. The present paper is intended to be a step toward a better understanding of the space.

It is easy to observe that the proofs of the above-mentioned known results concerning the spaces of harmonic maps share the following common technique: for an arbitrary harmonic map as above, there exists a smooth deformation given as the gradient flow of a Morse-Bott function over the corresponding twistor space. The deformation reduces the codimension in the target space while it respects the degree and the energy. By using the deformation, important information,such as the connectedness or the fundamental group, of the space $\operatorname{Harm}_{d}\left(S^{2}, M^{n_{1}}\right)$ of harmonic maps is obtained out of the information of the spaces $\operatorname{Harm}_{d}\left(S^{2}, M^{n_{2}}\right)\left(n_{1} \geq n_{2}\right)$

Received by the editors October 24, 1995 and, in revised form, February 1, 1996.

1991 Mathematics Subject Classification. Primary 49F10; Secondary 58E20.

Key words and phrases. Harmonic 2-spheres, extra eigenfunctions, null curves. 
of harmonic maps of lower codimension. In this way, an induction on codimension proceeds and we get the results mentioned above. We, however, need more detailed information on each step of deformation in order to understand the space $\operatorname{Harm}_{d}\left(S^{2}, M^{n_{1}}\right)$ better. In other words, we should know exactly which elements of $\operatorname{Harm}_{d}\left(S^{2}, M^{n_{2}}\right)$ come from the above deformation. Equivalently, we have to explicitly describe the subset of $\operatorname{Harm}_{d}\left(S^{2}, M^{n_{2}}\right)$ which consists of those deformed maps. In this paper we will study such subsets in the case of $\operatorname{Harm}_{d}\left(S^{2}, S^{n}\right)$.

Let $L_{r}$ be the space of harmonic maps $x \in \operatorname{Harm}_{d}\left(S^{2}, S^{n}\right)$ which admit smooth deformations $x_{t}: S^{2} \longrightarrow S^{n+2 r}$ of full harmonic maps of degree $d$, with $\lim _{t \rightarrow \infty} x_{t}=$ $x$. We will show the space $L_{r}$ is equal to the space $P_{r}$ of harmonic maps in $\operatorname{Harm}_{d}\left(S^{2}, S^{n}\right)$ which have exactly $r$ pairs of extra eigenfunctions (see $\S 1$ for the definition). This is conjectured by Ejiri and has been proved in the case of $r=1$ [E1], [E2]. The space $P_{r}$ was studied in [EK1], [EK2] through the study of the Gauss maps of the complete totally isotropic minimal surfaces in the Euclidean space with only planar ends. It was shown, among others, that $P=\bigcup_{r} P_{r}$ has an algebraic structure and that it has codimension greater than one. In this paper, we will reconstruct the space $\operatorname{Harm}_{d}\left(S^{2}, S^{n+2 r}\right)$ by the above deformations and the set $L_{r}=P_{r}$ of their limit maps.

The author would like to express her gratitude to the Max-Planck-Institut für Mathematik for its hospitality while this paper was written and to the referee for his useful suggestions.

\section{§1. Preliminaries}

Let $x: S^{2} \longrightarrow S^{n}$ be a full harmonic map from a compact Riemann surface $S^{2}$ of genus 0 to the unit sphere $S^{n}$ of degree $d$. Due to Calabi [Ca], the dimension $n$ of the target manifold $S^{n}$ is always even for a full harmonic map . From now on, we only consider a harmonic map $x: S^{2} \longrightarrow S^{2 m}$. Then $x$, as a $(2 m+1)$-vector valued function, satisfies

$$
\Delta x+2 x=0,
$$

where $\Delta$ is the Laplacian with respect to the metric induced by $x$. It implies that the $2 m+1$ coordinate functions of $x$ are eigenfunctions of $\Delta$ with eigenvalue 2 . In this paper, eigenfunctions always mean eigenfunctions of $\Delta$ with eigenvalue 2 . An eigenfunction is called an extra eigenfunction if it is not a linear combination of coordinate functions. In [EK2] it was proved that $x$ rarely has extra eigenfunctions. More precisely, we have

Theorem 1.1 ([EK2]). The set $P$ of all full harmonic maps $x: S^{2} \longrightarrow S^{2 m}$ which admits extra eigenfunctions has $\operatorname{codim}_{\mathbf{R}} \geqq 2$ in $\operatorname{Harm}_{d}\left(S^{2}, S^{2 m}\right)$ and has a certain algebraic structure.

We will quickly review in this section the results in [EK2] which we will need later.

Assume $x$ admits an extra eigenfunction $f$. Then we can construct a complete isotropic branched minimal surface $X$ in $\mathbf{R}^{2 m+1}$ with the total curvature $4 \pi d$, whose Gauss map is $x$ and $f=\langle X, x\rangle$. We can also show that $X$ has only planar ends. Actually there is a one-one correspondence between the set $P$ and the set of all complete isotropic branched minimal surfaces of genus zero with only planar ends. We obtain a constant map $X=A$ in $\mathbf{R}^{2 m+1}$ if we choose $f$ to be a coordinate function instead of an extra eigenfunction in the above construction. 
Proposition 1.2 ([EK2]). Let $X$ be a complete isotropic branched minimal surface in $\mathbf{R}^{2 m+1}$. Take an isothermal coordinate $z$ around a planar end $z=0$. Then $X_{z}$ has a Laurent expansion around the end $z=0$ such that

$$
X_{z}=\frac{1}{z^{k}} V_{-k}+\cdots+\frac{1}{z} V_{-1}+\text { holomorphic part }
$$

(1) $V_{-1}=0$,

(2) $\left\langle V_{-i}, V_{-j}\right\rangle=0 \quad$ for $2 \leq i, j \leq k$,

(3) $\left\langle V_{-i}, x\right\rangle=0 \quad$ for $2 \leq i \leq k$,

where $\langle$,$\rangle is the complex bi-linear form induced by the standard metric \langle$,$\rangle of$ $\mathbf{R}^{2 m+1}$.

The conjugate minimal surface $Y=\operatorname{Re}\left[\int \sqrt{-1} X_{z} d z\right]$ is well-defined, up to a constant, because of (1). Notice that $Y$ also has $x$ as its Gauss map and its support function $\langle Y, x\rangle$ satisfies

$$
\Delta\langle Y, x\rangle+2\langle Y, x\rangle=0
$$

As $Y$ is not a constant map, $\langle Y, x\rangle$ is not a linear combination of the coordinate functions of $x$. Hence, if $x$ has an extra eigenfunction $f=\langle X, x\rangle$, then it has one more extra eigenfunction $\langle Y, x\rangle$. We call them a pair of extra eigenfunctions.

Let $X$ be the full complete isotropic branched minimal surfaces in $\mathbf{R}^{2 m+1}$ constructed as above, $Y$ be its conjugate minimal surface and $z$ be a local isothermal coordinate. It is known that the trivial bundle $\underline{\mathbf{C}^{2 m+1}}=S^{2} \times \mathbf{C}^{2 m+1}$ decomposes orthogonally as

$$
\underline{\mathbf{C}^{2 m+1}}=W_{1}+\cdots+W_{m}+\bar{W}_{1}+\cdots+\bar{W}_{m}+\{x\},
$$

where each $W_{1}+\cdots+W_{k}$ is the holomorphic subbundle of rank $k$ locally spanned by $\left\{\frac{\partial X}{\partial z}, \ldots, \frac{\partial^{k} X}{\partial z^{k}}\right\}$ for $1 \leq k \leq m$. It should be remarked that the above decomposition does not depend on $X$ but on only $x$. Let $E_{k}$ be the local section of unit length generating $W_{k}$ (see [EK2] for details). Note that

$$
\left\{\frac{\partial Y}{\partial z}, \ldots, \frac{\partial^{k} Y}{\partial z^{k}}\right\} \cap\left\{\frac{\partial Y}{\partial z}, \ldots, \frac{\partial^{k-1} Y}{\partial z^{k-1}}\right\}^{\perp}, \quad 1 \leq k \leq m,
$$

is also generated by the same $E_{k}$.

Lemma 1.3. Let $E_{k}$ be as above. Then

(1) $\left\langle E_{i}, E_{j}\right\rangle=0 \quad$ for $1 \leq i, j \leq m$,

(2) $\left\langle E_{i}, \bar{E}_{j}\right\rangle=\delta_{i, j}$ for $1 \leq i, j \leq m$,

(3) $\left\langle E_{i}, x\right\rangle=0 \quad$ for $1 \leq i \leq m$.

We call $\left\{E_{1}, \ldots, E_{m}\right\}$ the standard isotropic basis corresponding to $x$. Let $G=$ $X+\sqrt{-1} Y$. Then $G: S^{2} \longrightarrow \mathbf{C}^{2 m+1}$ is a meromorphic null curve, i.e.,

$$
\begin{gathered}
\bar{\partial} G=0, \\
\left\langle\frac{\partial^{k} G}{\partial z^{k}}, \frac{\partial^{k} G}{\partial z^{k}}\right\rangle=0, \quad 1 \leq k \leq m .
\end{gathered}
$$

We call $\langle G, x\rangle=\langle X, x\rangle+\sqrt{-1}\langle Y, x\rangle$ a pair of extra eigenfunctions as well.

Note

$$
\left\{\frac{\partial G}{\partial z}, \ldots, \frac{\partial^{k} G}{\partial z^{k}}\right\}=W_{1}+\cdots+W_{k}, \quad 1 \leq k \leq m .
$$


Define $G_{+} \in\left\{W_{1}, \ldots, W_{m}\right\}$ and $G_{-} \in\left\{\bar{W}_{1}, \ldots, \bar{W}_{m}\right\}$ such that $G=G_{+}+$ $\langle G, x\rangle x+G_{-}$.

\section{Lemma 1.4.}

$$
\begin{gathered}
\partial G_{+}+h_{z} x \| E_{1}, \\
\partial G_{-}+h \partial x=0, \\
\bar{\partial} G_{+}+h \bar{\partial} x=0, \\
\bar{\partial} G_{-}+h \bar{z} x=0,
\end{gathered}
$$

where $h=\langle G, x\rangle$.

\section{§2. Deformations}

Let $x: S^{2} \longrightarrow S^{2 m}$ be a full harmonic map from $S^{2}$ to $S^{2 m}$ of degree $d$ with extra eigenfunctions $f_{1}, \ldots, f_{r}$. As in $\S 1$, we construct a complete isotropic branched minimal surface $X_{i}$ in $\mathbf{R}^{2 m+1}$, whose Gauss map is $x$ and support function $\left\langle X_{i}, x\right\rangle=f_{i}$, for $1 \leq i \leq r$. Let $Y_{i}$ be its conjugate and we define $G_{i}=X_{i}+\sqrt{-1} Y_{i}$ and $h_{i}=\left\langle G_{i}, x\right\rangle$. It is easy to see

\section{Lemma 2.1.}

$$
\begin{gathered}
\frac{\partial G_{i}}{\partial \bar{z}}=0 \\
\left\langle\frac{\partial^{k} G_{i}}{\partial z^{k}}, \frac{\partial^{k} G_{i}}{\partial z^{k}}\right\rangle=0, \quad 1 \leq k \leq m .
\end{gathered}
$$

We will construct a smooth deformation $x_{t}: S^{2} \longrightarrow S^{2(m+r)}$ of harmonic map of degree $d$ such that

(1) $\lim _{t \rightarrow \infty} x_{t}=x$,

(2) $x_{t}$ is full in $S^{2(m+r)}$ for $t \neq \infty$,

when no linear combination of the $h_{i}$ 's is equal to a linear combination of coordinate functions.

Let $e_{1}, \ldots, e_{2 r}$ be the standard orthonormal basis of $\mathbf{R}^{2 r}$ and let

$$
E_{m+i}=\frac{e_{2 i-1}-\sqrt{-1} e_{2 i}}{\sqrt{2}} .
$$

We may assume that the image of $x$ is contained in $S^{2(m+r)} \cap\left\{e_{1}, \ldots, e_{2 r}\right\}^{\perp}=S^{2 m}$. Take the standard isotropic basis (defined in $\S 1$ ) $E_{1}, \ldots, E_{m}$ corresponding to $x$. Note that $\frac{\partial^{k} G_{i}}{\partial z^{k}} \in \operatorname{span}\left\{E_{1}, \ldots, E_{k}\right\}$ for all $1 \leq i \leq r$ and $1 \leq k \leq m$.

Lemma 2.2. $c_{i, j}=\int\left\langle d G_{i}, G_{j}\right\rangle$ is well-defined for all $1 \leq i, j \leq m$.

Proof.

$$
\begin{gathered}
\left\langle d G_{i}, G_{j}\right\rangle=\left\langle\partial G_{i}, G_{j}\right\rangle d z=\left\langle\partial G_{i}, G_{j-}\right\rangle d z \\
=\partial\left\langle G_{i}, G_{j-}\right\rangle d z-\left\langle G_{i}, \partial G_{j-}\right\rangle d z \\
=d\left\langle G_{i}, G_{j-}\right\rangle-\bar{\partial}\left\langle G_{i}, G_{j-}\right\rangle d \bar{z}+\left\langle G_{i}, h_{j} \partial x\right\rangle d z \\
=d\left\langle G_{i}, G_{j-}\right\rangle+h_{i z} h_{j} d z+h_{i} h_{j \bar{z}} d \bar{z} .
\end{gathered}
$$


Therefore

$$
c_{i, j}=\left\langle G_{i}, G_{j-}\right\rangle+\int \omega,
$$

where $\omega=h_{i z} h_{j} d z+h_{i} h_{j \bar{z}} d \bar{z}$ is an exact form.

As both $h_{i}$ and $h_{j}$ are smooth functions on $S^{2}$, the second term on the right hand side of the last equation is well-defined and hence so is the left hand side.

Put $\tilde{G}_{i}=G_{i}+A_{i}$, where $A_{i}$ is a constant vector in $\mathbf{C}^{2 m+1}$. The integral $\tilde{c}_{i, j}=\int\left\langle d \tilde{G}_{i}, \tilde{G}_{j}\right\rangle$ is also well-defined. Define $F_{i}=\bar{E}_{m+i}+\tilde{G}_{i}-\sum_{j=1}^{r} \tilde{c}_{i, j} E_{m+j}$.

Remark. We can choose all the $A_{i}$ 's to be zero to construct a smooth deformation satisfying the above conditions (1) and (2). In $\S 3$ we will see what elements in $\operatorname{Harm}_{d}\left(S^{2}, S^{2 m+2 r}\right)$ can be deformed to a given $x$ in $\operatorname{Harm}_{d}\left(S^{2}, S^{2 m}\right)$. For that we take arbitrary constant vectors as $A_{i}$ 's.

Lemma 2.3. There are meromorphic functions $b_{1}, \ldots, b_{r}$ such that

$$
\Psi^{(k)}=\sum_{i=1}^{r} b_{i}^{(k)} F_{i}, \quad 0 \leq k \leq r-1,
$$

where $(k)$ stands for the $k$-th derivative by $z$.

Proof. It is enough to find meromorphic functions $b_{1}, \ldots, b_{r}$ such that

$$
\sum_{i=1}^{r} b_{i}^{(k)} F_{i}^{\prime}=\sum_{i=1}^{r} b_{i}^{(k)}\left\{G_{i}^{\prime}-\sum_{j=1}^{r}\left\langle G_{i}^{\prime}, \tilde{G}_{j}\right\rangle E_{m+j}\right\}=0, \quad 0 \leq k \leq r-2 .
$$

Recall that all the $G_{i}^{\prime}$ 's are parallel and define functions $\mu_{i}$ by $G_{i}^{\prime}=\mu_{i} E_{1}$. Let $\mathbf{b}=\left(b_{1}, b_{2}, \cdots, b_{r}\right)$, let $\mathbf{m}=\left(\mu_{1}, \mu_{2}, \cdots, \mu_{r}\right)$, and let $\mathbf{M}=\left(\mathbf{M}_{1},{ }^{t} \mathbf{m}^{(r-1)}\right)$ $=\left({ }^{t} \mathbf{m},{ }^{t} \mathbf{m}^{\prime}, \ldots,{ }^{t} \mathbf{m}^{(r-1)}\right)$ be the Wronskian matrix of $\mu_{1}, \mu_{2}, \cdots, \mu_{r}$. Take $\mathbf{b}$ as a solution of the equation $\mathbf{b M}_{1}=0$ and let $\psi=\mathbf{b}^{t} \mathbf{m}^{(r-1)}$. Then $b_{i}$ 's satisfy $\sum_{i=1}^{r} b_{i} \mu_{i}^{(k)}=0$ for $0 \leq k \leq r-2$. Since the conditions $\sum_{i=1}^{r} b_{i}^{(k)} \mu_{i}=0$ for $0 \leq k \leq$ $r-2$ are equivalent to the conditions $\sum_{i=1}^{r} b_{i} \mu_{i}^{(k)}=0$ for $0 \leq k \leq r-2$, it is easy to see that those $b_{i}$ 's satisfy $\sum_{i=1}^{r} b_{i}^{(k)} G_{i}^{\prime}=\sum_{i=1}^{r} b_{i}^{(k)} \mu_{i} E_{1}=0$ for $0 \leq k \leq r-2$. That proves that $\sum_{i=1}^{r} b_{i}^{(k)} F_{i}^{\prime}=0$ for those $b_{i}$ 's. Since $\bar{\partial} \log \mu_{1}=\bar{\partial} \log \mu_{2}=\cdots=\bar{\partial} \log \mu_{r}$ $\left(=\bar{\alpha}_{1}\right.$ in Lemma 1.3 in [EK2]), $\mathbf{b} \bar{\partial} \mathbf{M} \| \mathbf{b}$. We can choose $\psi$ so that $b_{i}$ 's are meromorphic functions.

Remark. The $\Psi=\sum_{i=1}^{r} b_{i} F_{i}$ may not be full if we take $b_{1}, \cdots, b_{r}$, whose existence is assured in Lemma 2.3, at random. Later (in Lemma 2.5), we will see when $b_{1}, \cdots, b_{r}$ are independent.

Lemma 2.4. $\Psi=\sum_{i=1}^{r} b_{i} F_{i}: S^{2} \longrightarrow \mathbf{C}^{2(m+r)+1}$ is a totally isotropic meromorphic curve, i.e.,

$$
\left\langle\frac{\partial^{k} \Psi}{\partial z^{k}}, \frac{\partial^{k} \Psi}{\partial z^{k}}\right\rangle=0, \quad 0 \leq k \leq m+r-1
$$

Proof. From

$$
\left\langle F_{i}, F_{j}\right\rangle=-\tilde{c}_{i, j}-\tilde{c}_{j, i}+\left\langle\tilde{G}_{i}, \tilde{G}_{j}\right\rangle=0,
$$

it follows that $\left\langle\Psi^{(k)}, \Psi^{(k)}\right\rangle=0$ for $0 \leq k \leq r-1$. $\left\langle\Psi^{(r+k)}, \Psi^{(r+k)}\right\rangle=0$ for $0 \leq k \leq m-1$ because $F_{i}^{(k)}=G_{i}^{(k)}-\sum_{j=1}^{r}\left\langle G_{i}^{(k)}, G_{j}\right\rangle E_{m+j}$ is a null vector and $\left\langle F_{i}^{(k)}, F_{j}^{(l)}\right\rangle=0$ for $1 \leq k \leq m, 1 \leq l \leq m$. 
Before we construct the deformation, we briefly review Calabi's twistor construction $[\mathrm{Ca}]$. Let $Z_{n}=\left\{W: n\right.$-dimensional complex subspaces in $\mathbf{C}^{2 n+1}, W \perp$ $\bar{W}\}=S O(2 n+1) / U(n)$ and call $Z_{n}$ the twisor space. Define the projection $\pi: Z_{n} \longrightarrow S^{2 n}$ by $\pi(W)=\{W \bigoplus \bar{W}\}_{\mathbf{R}}^{\perp}$. More precisely $x=\pi(W)$ is a unit vector $x \in S^{2 n}$ such that the orientation of $\{W \bigoplus \bar{W}\}_{\mathbf{R}} \bigoplus\{x\}$ agrees with the natural orientation of $\mathbf{R}^{2 n+1}$ and $\pi^{-1}(x)=\left\{\frac{\partial x}{\partial z} \wedge \cdots \wedge \frac{\partial^{n} x}{\partial z^{n}}\right\}$. There is the so-called Calabi 2:1 correspondence between the space $\operatorname{Harm}_{d}^{\text {full }}\left(S^{2}, S^{2 n}\right)$ of full harmonic maps in $\operatorname{Harm}_{d}\left(S^{2}, S^{2 n}\right)$ and the space $H H_{d}^{f u l l}\left(S^{2}, Z_{n}\right)$ of full holomorphic maps from $S^{2}$ to $Z_{n}$, that are horizontal with respect to the projection $\pi$. This 2:1 correspondence arises because both $x$ and $-x$ are harmonic maps in $S^{2 n}$. We call $x$ a harmonic map with natural orientation and $-x$ a harmonic map with reverse orientation and denote by $\operatorname{Harm}_{d}^{ \pm}\left(S^{2}, S^{2 n}\right) \subset \operatorname{Harm}_{d}\left(S^{2}, S^{2 n}\right)$ the space of harmonic maps with natural orientation and the space of harmonic maps with reverse orientation, respectively. $\operatorname{Harm}_{d}\left(S^{2}, S^{2 n}\right)=\operatorname{Harm}_{d}^{+}\left(S^{2}, S^{2 n}\right) \cup$ $\operatorname{Harm}_{d}^{-}\left(S^{2}, S^{2 n}\right), \operatorname{Harm}_{d}^{\text {nonfull }}\left(S^{2}, S^{2 n}\right)=\operatorname{Harm}_{d}^{+}\left(S^{2}, S^{2 n}\right) \cap \operatorname{Harm}_{d}^{-}\left(S^{2}, S^{2 n}\right)$ and $\operatorname{Harm}_{d}^{+}\left(S^{2}, S^{2 n}\right) \simeq \operatorname{Harm}_{d}^{-}\left(S^{2}, S^{2 n}\right)$. (See [FGKO] for details.) In this paper, we mainly look at the space $\operatorname{Harm}_{d}^{+}\left(S^{2}, S^{2 n}\right)$ rather than $\operatorname{Harm}_{d}\left(S^{2}, S^{2 n}\right)$, but abuse the notation.

Now we consider an action $A_{t} \in S O(2 m+2 r+1, \mathbf{C})$ on $Z_{m+r}$ defined by

$$
\left\{\begin{aligned}
A_{t} E_{m+i} & =e^{-t} E_{m+i}, \quad 1 \leq i \leq r \\
A_{t} \bar{E}_{m+i} & =e^{t} \bar{E}_{m+i}, \quad 1 \leq i \leq r \\
A_{t} & =\mathrm{id} \quad \text { on the orthogonal complement. }
\end{aligned}\right.
$$

The action $A_{t}$ induces a deformation in $H H_{d}\left(S^{2}, Z_{m+r}\right)$, since it preserves holomorphicity and horizontality of maps. By the similar argument in [FGKO], we can see this deformation is a gradient flow of a Morse-Bott function over $Z_{m+r}$ and its critical manifolds are

$$
\begin{aligned}
C_{k, l} & =\left\{W_{0} \oplus W_{1} \oplus W_{2} \in Z_{m+r}: W_{0} \text { is an } m\right. \text {-dimensional subspace in } \\
& \left\{E_{m+1}, \cdots, E_{m+r}, \cdots \bar{E}_{m+1}, \cdots, \bar{E}_{m+r}\right\}^{\perp}, W_{0} \perp \bar{W}_{0}, \\
& W_{1} \text { is a } k \text {-dimensional subspace in }\left\{E_{m+1}, \cdots, E_{m+r}\right\}, W_{1} \perp \bar{W}_{1}, \\
& \left.W_{2} \text { is an } l \text {-dimensional subspace in }\left\{\bar{E}_{m+1}, \cdots, \bar{E}_{m+r}\right\}, W_{2} \perp \bar{W}_{2}\right\},
\end{aligned}
$$

for $k+l=r$. Notice that $C_{0, r}, C_{r, 0} \cong Z_{m}$ and

$$
\pi\left(C_{k, l}\right) \subset S^{2 m+2 r} \cap\left\{E_{m+1} \wedge \cdots \wedge E_{m+r} \wedge \cdots \wedge \bar{E}_{m+1} \wedge \cdots \wedge \bar{E}_{m+r}\right\}^{\perp}=S^{2 m} .
$$

Now we have an element $\Omega=\Psi \wedge \Psi^{\prime} \wedge \ldots \wedge \Psi^{(r+m-1)}$ in $H H_{d}\left(S^{2}, Z_{r+m}\right)$ for the totally isotropic meromorphic curve $\Psi$ in Lemma 2.4. We apply the above action $A_{t}$ to $\Omega$ and get a 1-parameter smooth deformation

$$
\Omega_{t}=\Psi_{t} \wedge \Psi_{t}^{\prime} \wedge \ldots \wedge \Psi_{t}^{(r+m-1)},
$$

where the meromorphic null map

$$
\Psi_{t}=\sum_{i=1}^{r} b_{i} F_{i_{t}}=\sum_{i=1}^{r} b_{i}\left\{e^{t} \bar{E}_{m+i}+\tilde{G}_{i}-\sum_{j=1}^{r} \tilde{c}_{i, j} e^{-t} E_{m+j}\right\} .
$$

By an easy calculation, we get 
Lemma 2.5.

$$
\begin{gathered}
\Omega_{t}=\Psi_{t} \wedge \Psi_{t}^{\prime} \wedge \ldots \wedge \Psi_{t}^{(r+m-1)} \\
=B\left(\sum_{k} b_{k}^{(r-1)} \mu_{k}\right)^{m} F_{1} \wedge F_{2} \wedge \cdots \wedge F_{r} \wedge F_{r+1} \wedge \cdots \wedge F_{r+m},
\end{gathered}
$$

where

$$
\begin{gathered}
B=\operatorname{det}\left|\begin{array}{llll}
b_{1} & b_{2} & \ldots & b_{r} \\
b_{1}^{\prime} & b_{2}^{\prime} & \ldots & b_{r}^{\prime} \\
\vdots & \vdots & \vdots & \vdots \\
b_{1}^{(r-1)} & b_{2}^{(r-1)} & \ldots & b_{r}^{(r-1)}
\end{array}\right|, \\
F_{r+k}=\beta_{1} \ldots \beta_{k-1}\left(\bar{E}_{r+k}-\sum_{j=1}^{r}\left\langle E_{r+k}, G_{j}\right\rangle e^{-t} E_{m+j}\right) \\
=\frac{F_{i}^{(k)}}{\mu_{k}} \cap\left\{F_{i}^{\prime}, \ldots, F_{i}^{(k-1)}\right\}^{\perp} .
\end{gathered}
$$

If and only if no linear combination of $h_{1}, \ldots h_{r}$ is a linear combination of coordinate functions, we can take the $b_{i}$ 's so that $B \neq 0$.

Proof. We prove only the last assertion. Let us use the same notation as in the proof of Lemma 2.3. It is known that meromorphic functions $b_{1}, \ldots, b_{r}$ are linearly dependent if and only if the Wronskian $B=0$. Recall $b_{i}$ 's are determined by the equations $\sum_{i=1}^{r} \mu_{i}^{(k)} b_{i}=0$ for $0 \leq k \leq r-2$, i.e., $\mathbf{b}$ is perpendicular to the vectors $\mathbf{m}, \ldots, \mathbf{m}^{(r-2)}$. When $b_{1}, \ldots, b_{r}$ are linearly dependent, this means that a linear combination of $\mathbf{m}, \ldots, \mathbf{m}^{(r-2)}$ is a non-zero constant vector. Thus $\mu_{1}, \ldots, \mu_{r}$ are solutions of a homogeneous linear O.D.E. of order $r-1$, i.e., there are constants $c_{i}$ such that $\sum_{i=1}^{r} c_{i} \mu_{i}=0$. On the other hand, if $\sum_{i=1}^{r} c_{i} \mu_{i}=0$ for some constants $c_{1}, \ldots, c_{r}$, we can choose $\mathbf{b}=\left(c_{1}, c_{2}, \ldots, c_{r}\right)$. For this $\mathbf{b}$, the Wronskian $B=0$. Thus we prove that $\sum_{i=1}^{r} c_{i} \mu_{i}=0$ for some constants $c_{i}$ if and only if $B=0$ for some $\mathbf{b}$. When $\sum_{i=1}^{r} c_{i} \mu_{i}=0$ for some constants $c_{i}, \sum_{i=1}^{r} c_{i} G_{i}$ is a constant vector $A$ in $\mathbf{C}^{2 m+1}$, because

$$
\begin{gathered}
\partial\left(\sum_{i=1}^{r} c_{i} G_{i}\right)=\sum_{i=1}^{r} c_{i} \partial G_{i}=\left(\sum_{i=1}^{r} c_{i} \mu_{i}\right) E_{1}=0, \\
\bar{\partial}\left(\sum_{i=1}^{r} c_{i} G_{i}\right)=\sum_{i=1}^{r} c_{i} \bar{\partial} G_{i}=0 .
\end{gathered}
$$

We can see that $\sum_{i=1}^{r} c_{i} h_{i}=\left\langle\sum_{i=1}^{r} c_{i} G_{i}, x\right\rangle=\langle A, x\rangle$ is a linear combination of coordinate functions.

We say that extra eigenfunctions $h_{1}, \ldots h_{r}$ are linearly independent when no linear combination of $h_{1}, \ldots h_{r}$ is a linear combination of coordinate functions. As we see in Lemma 2.5, $\Omega_{t} \in H H^{\text {full }}\left(S^{2}, Z_{r+m}\right)$ for $t \neq \infty$ when $h_{1}, \ldots h_{r}$ are linearly independent and therefore we can define $x_{t}=\pi\left(\Omega_{t}\right) \in \operatorname{Harm}\left(S^{2}, S^{2 r+2 m}\right)$.

Proposition 2.6. If $h_{1}, \ldots, h_{r}$ are linearly independent, then $x_{t}$ constructed above satisfies 
(1) For $t \neq \infty, x_{t}=\left\{\Omega_{t} \wedge \bar{\Omega}_{t}\right\}^{\perp}$ defines a full harmonic map from $S^{2}$ to $S^{2(r+m)}$,

(2) $\lim _{t \rightarrow \infty} x_{t}=x$,

(3) $x_{t}$ preserves degree $d$.

Proof. $\Omega_{t}$ is spanned by $F_{1_{t}}, \ldots, F_{r+m_{t}}$. We will find a better basis. Let $F_{r+i_{t}}^{*}=$ $E_{r+i}-\sum_{j=1}^{r}\left\langle E_{r+i}, G_{j}\right\rangle e^{-t} E_{m+j}$ for $1 \leq i \leq m$ and $F_{i_{t}}^{*}=F_{i_{t}}-$ $\sum_{j=1}^{m}\left\langle G_{i}, \bar{E}_{j}\right\rangle F_{r+j_{t}}^{*}=e^{t} \bar{E}_{m+i}+\left(G_{i}-G_{i+}\right)-\sum_{j=1}^{r} \int h_{i z} h_{j} d z e^{-t} E_{m+j}$ for $1 \leq i \leq r$. We see that $G_{i}-G_{i+}=G_{i-}+h_{i} g$ satisfies

$$
\begin{aligned}
\partial\left(G_{i-}+h_{i} g\right) & =\partial h_{i} x, \\
\bar{\partial}\left(G_{i-}+h_{i} g\right) & =h_{i} \bar{\partial} x .
\end{aligned}
$$

Since $x$ is a harmonic map on $S^{2}$ and $\operatorname{Re}\left(h_{i}\right)$ and $\operatorname{Im}\left(h_{i}\right)$ are eigenfunctions of $\Delta$ with respect to the metric induced by $x$, the above equations show that $G_{i}-G_{i+}=$ $G_{i-}+h_{i} x$ is bounded. We use the basis $\left\{F_{1_{t}}^{*}, \ldots, F_{r_{t}}^{*}, F_{r+1_{t}}^{*}, \ldots, F_{r+m_{t}}^{*}\right\}$. It is easy to see that $\Omega_{t}$ does not intersect with any critical manifold $C_{k, l}$ for $k+l=r$, which proves that the deformation constructed preserves the degree. By an easy calculation, we get

$$
\begin{gathered}
x_{t}=\left\{\Omega_{t} \wedge \bar{\Omega}_{t}\right\}^{\perp} \\
=x+e^{-t} \sum_{i=1}^{r}\left(\bar{h}_{i} E_{m+i}+h_{i} \bar{E}_{m+i}\right)+o\left(e^{-2 t}\right) .
\end{gathered}
$$

As $t$ goes to $\infty, x_{t}$ converges to $x$. Assume $x_{t}, t \neq \infty$, is not full. Then $\sum_{i=1}^{r}\left(\bar{h}_{i} E_{m+i}+h_{i} \bar{E}_{m+i}\right)$ must lie in a hyperplane of $\operatorname{span}\left\{E_{m+1}, \ldots, E_{m+r}, \bar{E}_{m+1}\right.$, $\left.\ldots, \bar{E}_{m+r}\right\}$. Because the eigenfunctions $h_{1}, \ldots, h_{r}$ are linearly independent, this means that $\bar{h}_{i} E_{m+i}+h_{i} \bar{E}_{m+i}$ is parallel to a constant line for some $i$, which is impossible. Hence we have proved that $x_{t}$ is full for all $t \neq \infty$.

We have

Theorem A. Suppose a full harmonic map $x: S^{2} \longrightarrow S^{2 m}$ of degree $d$ has $r$ linearly independent pairs of extra eigenfunctions. We can construct a smooth deformation of full harmonic maps $x_{t}: S^{2} \longrightarrow S^{2(r+m)}$, preserving degree $d$ and converging to $x$ as $t$ goes to $\infty$.

Corollary. The space $P_{r}$ of full harmonic maps $x: S^{2} \longrightarrow S^{2 m}$ of degree $d$ which admit $r$ linearly independent pairs of extra eigenfunctions is equal to the space $L_{r}$ of full harmonic maps $x: S^{2} \longrightarrow S^{2 m}$ of degreed which admit the smooth deformations as in Theorem A.

Proof. We have seen that $x \in P_{r}$ admits such a deformation. On the other hand, suppose that $x=\lim _{t \rightarrow \infty} x_{t}$. Then $x_{t}$ has at least $2(m+r)+1$ eigenfunctions of eigenvalue 2 as its coordinate functions. Because of the continuity of $\Delta, x$ also must have at least $2(r+m)+1$ eigenfunctions. However, $x$ has only $2 m+1$ linearly independent coordinate functions and hence $x$ has $2 r$ linearly independent extra eigenfunctions.

Corollary. A harmonic map $x: S^{2} \longrightarrow S^{2 m}$ of degree $d$ has at most $\sqrt{8 d+1}-$ $2 m-1$ linearly independent extra eigenfunctions. In particular, $x$ has no extra eigenfunction when $d=\frac{m(m+1)}{2}$. 
Proof. If $x$ has $r$ linearly independent pairs of extra eigenfunctions, then we construct a full harmonic maps $x_{t}: S^{2} \longrightarrow S^{2 m+2 r}$ of degree $d$. Due to [B], $d \geq$ $\frac{(m+r)(m+r+1)}{2}$.

\section{§3.DIMENSION}

Lemma 3.1. Let $V_{x}$ be the set of harmonic maps in $\operatorname{Harm}_{d}\left(S^{2}, S^{2 m+2 r}\right)$ which can be deformed to a fixed element $x \in \operatorname{Harm}_{d}\left(S^{2}, S^{2 m}\right)$. Then the set $\bigcup_{x \in L_{r}} V_{x}$ is open dense in $\operatorname{Harm}_{d}\left(S^{2}, S^{2(m+r)}\right)$.

Proof. Take a harmonic map $y \in \operatorname{Harm}_{d}\left(S^{2}, S^{2(m+r)}\right)$ and $y_{t}=A_{t} y$. This $y_{t}$ gives a deformation such that $\lim _{t \rightarrow \infty} y_{t}=t_{\infty}: S^{2} \longrightarrow S^{2 m}$. If the image of the corresponding holomorphic horizontal curve in $Z_{m+r}$ of $y$ does not intersect with any of the critical manifolds $C_{k, l}$ for $k+l=r$, the deformation preserves the degree and $y$ admits a deformation $y_{t}$ which converges to an element $y_{\infty}$ of $\operatorname{Harm}_{d}\left(S^{2}, S^{2 m}\right)$ as $t$ goes to $\infty$. Notice that

$$
\begin{aligned}
& \operatorname{dim} Z_{m+r}-\max _{k+l=r} \operatorname{dim} C_{k, l}-2-1 \\
& =(m+r)(m+r+1)-\max _{k+l=r}\{2 k l+m(m+1)\}-3 \\
& \qquad\left\{\begin{array}{l}
\geq \frac{r^{2}}{2}+r(2 m+1)-3 \geq 0, \quad 2 \leq r, \\
\geq 2 m-1 \geq 0, \quad r=1,
\end{array}\right.
\end{aligned}
$$

which implies that a generic $y \in \operatorname{Harm}_{d}\left(S^{2}, S^{2(m+r)}\right)$ can be deformed to an element $y_{\infty} \in \operatorname{Harm}_{d}\left(S^{2}, S^{2 m}\right)$.

Lemma3.2. For an arbitrary $x \in L_{r} \subset \operatorname{Harm}_{d}^{f u l l}\left(S^{2}, S^{2 m}\right)$, the dimension of the set $V_{x}$ of harmonic maps in $\operatorname{Harm}_{d}^{\text {full }}\left(S^{2}, S^{2 m+2 r}\right)$ which can be deformed to $x$ is $r^{2}+r(2 m+1)$.

Proof. If there is such a deformation $x_{t}$ with $x=\lim _{t \rightarrow \infty} x_{t}$, we can take the following basis of the twistor lift $W_{t}$ of $x_{t}$ :

$$
W_{t}=\operatorname{span}\left\{\begin{array}{ccc}
e^{t} \bar{E}_{m+1}+G_{1}+e^{-t} u_{1}, & \ldots, & e^{t} \bar{E}_{m+r}+G_{r}+e^{-t} u_{r}, \\
w_{1}+e^{-t} u_{r+1}, & \ldots, & w_{m}+e^{-t} u_{r+m}
\end{array}\right\},
$$

where all $G_{i}$ 's are meromorphic curves in $\mathbf{C}^{2 m+1}$, all $u_{i}$ 's take value in the space spanned by $E_{m+1}, \ldots, E_{m+r}$, and $\left\{w_{1}, \ldots, w_{m}\right\}$ is a basis of the twistor lift $W_{\infty}$ of $x$ in $\mathbf{C}^{2 m+1}$. From the holomorphic horizontal conditions for $W_{t}$, we can see that $G_{i}$ 's are meromorphic null curves, and $u_{i}=-\sum_{k=1}^{r} c_{i, k} E_{m+k}$ with $\partial c_{i, k}=\left\langle\partial G_{i}, G_{k}\right\rangle$ for $1 \leq i \leq r$ and $u_{r+j}=-\sum_{k=1}^{r}\left\langle w_{j}, G_{k}\right\rangle E_{m+k}$ for $1 \leq j \leq m$.

We can also see that $\left\langle G_{i}, x\right\rangle$ 's are extra eigenfunctions if $x_{t}$ is full. Thus if there is such a deformation $x_{t}$, it is given in the form we have constructed in Theorem A. $G_{i}$ is uniquely determined up to constant multiple, a constant vector $A_{i}$ and a choice of a unitary basis $E_{m+1}, \ldots, E_{m+r}$. The dimension of $V_{x}$ is $r^{2}+r(2 m+1)$.

Corollary. We have

$$
\begin{aligned}
& \operatorname{dim} \operatorname{Harm}_{d}\left(S^{2}, S^{2(m+r)}\right) \\
& \quad=\operatorname{dim} \operatorname{Harm}_{d}\left(S^{2}, S^{2 m}\right)+\left(r-\operatorname{codim} L_{r}\right)+(r+m)^{2}-m^{2},
\end{aligned}
$$

and codim $L_{r}-r$ does not depend on $r$. 
Remark. If we prove $\operatorname{codim} L_{r}=r$ for some $r$, then we prove $\operatorname{dim} \operatorname{Harm}_{d}\left(S^{2}, S^{2 N}\right)=$ $2 d+N^{2}$, which is a conjecture in [V] (See [BW1], [BW2] for more about the moduli space of harmonic 2 -spheres.)

Proof. By Lemma 3.1, the set $\bigcup_{x \in L_{r}} V_{x}$ is open dense in $\operatorname{Harm}_{d}\left(S^{2}, S^{2(m+r)}\right)$. Thus we obtain

$$
\begin{aligned}
& \operatorname{dim} \operatorname{Harm}_{d}\left(S^{2}, S^{2(m+r)}\right)=\operatorname{dim} \bigcup_{x \in L_{r}} V_{x}=\operatorname{dim} L_{r}+\operatorname{dim} V_{x} \\
= & \operatorname{dim} \operatorname{Harm}_{d}\left(S^{2}, S^{2 m}\right)-\operatorname{codim} L_{r}+r^{2}+r(2 m+1) \\
= & \operatorname{dim} \operatorname{Harm}_{d}\left(S^{2}, S^{2 m}\right)+\left(r-\operatorname{codim} L_{r}\right)+(r+m)^{2}-m^{2} .
\end{aligned}
$$

\section{REFERENCES}

[B] J.L.M.Barbosa, On minimal immersions of $S^{2}$ into $S^{2 m}(1)$, Trans.Amer.Math.Soc. 210 (1975), 75-105. MR 51:11362

[BW1] J.Bolton and L.M.Woodward, Moduli spaces of harmonic 2-spheres, Geometry and Topology of Submanifolds IV, World Scientific, 1992, pp. 143-151. MR 93g:58035

[BW2] J.Bolton and L.M.Woodward, The space of harmonic maps of $S^{2}$ into $S^{n}$, Geometry and Global Analysis, Report of the first MSJ International Research Institute, Tôhoku University, 1993, pp. 165-173. MR 96k:58053

[Ca] E. Calabi, Minimal immersions of surfaces in Euclidean spheres, J. Diff. Geom. 1 (1976), 111-125. MR 38:1616

[Cr] T.A.Crawford, The space of harmonic maps from the 2-spheres to complex projective space, preprint, McGill University., 1993.

[E1] N.Ejiri, Minimal deformation of a nonfull minimal surface in $S^{4}(1)$, Compositio.Math. 90 (1994), 183-209. MR 95a:53098

[E2] N.Ejiri, The boundary of the space of full harmonic maps of $S^{2}$ into $S^{2 m}(1)$ and extra eigenfunctions, in preparation.

[EK1] N.Ejiri and M.Kotani, Index and flat ends of minimal surfaces, Tokyo J. Math. 16 (1993), 37-48. MR 94g:53003

[EK2] N.Ejiri and M.Kotani, Minimal surfaces in $S^{2 m}(1)$ with extra eigenfunctions, Quart. J. Math. 43 (1992), 421-440. MR 93k:53061

[FGKO] M.Furuta, M.A.Guest, M.Kotani and Y.Ohnita, On the fundamental group of the space of harmonic 2-spheres in the n-sphere, Math. Z. 215 (1994), 503-518. MR 95e:58047

[GMO] M.A.Guest, M.Mukai and Y.Ohnita, On the topology of spaces of harmonic 2-spheres in symmetric spaces, in preparation.

[GO] M.A.Guest and Y.Ohnita, Group actions and deformation for harmonic maps, J. Math. Soc. Japan 45 (1993), 671-704. MR 94m:58058

[K] M.Kotani, Connectedness of the space of minimal 2-spheres in $S^{2 m}(1)$, Proc. Amer. Math. Soc. 120 (1994), 803-810. MR 94e:58033

[L] B.Loo, The space of harmonic maps of $S^{2}$ into $S^{4}$, Trans. Amer. Math. Soc. 313 (1989), 81-103. MR 90k:58050

[M1] M.Mukai, On connectedness of the space of harmonic 2-spheres in quaternionic projective spaces, to appear, Tokyo J.Math..

[M2] M.Mukai, On connectedness of the space of harmonic 2-spheres in real Grassmann manifolds of 2-planes, Natur.Sci.Reo.Ochanomizu Univ. 44 (1993), 99-115. MR 95a:58031

[V] J.L.Verdier, Two dimensional $\sigma$ models and harmonic maps from $S^{2}$ to $S^{2 n}$, Lecture Notes in Physics, vol. 180, Berlin, Springer, 1983, p. 136-141.

Department of Mathematics, Faculty of Sciences, Toho University, Funabashi, Chiba, 274 , JAPAN

E-mail address: kotani@tansei.cc.u-tokyo.ac.jp 